\title{
Screening for tuberculosis and prediction of disease in Portuguese healthcare workers
}

José Torres Costa ${ }^{1,2}$, Rui Silva ${ }^{1,2}$, Felix C Ringshausen ${ }^{3}$ and Albert Nienhaus ${ }^{3^{*}}$

\begin{abstract}
Introduction: Results of systematic screening of healthcare workers (HCWs) for tuberculosis (TB) with the tuberculin skin test (TST) and interferon- $\gamma$ release assays (IGRA) in a Portuguese hospital from 2007 to 2010 are reported.

Methods: All HCWs are offered screening for TB. Screening is repeated depending on risk assessment. TST and QuantiFERON Gold In-Tube (QFT) are used simultaneously. X-ray is performed when TST is $>10 \mathrm{~mm}$, IGRA is positive or typical symptoms exist.

Results: The cohort comprises 2,889 HCWs. TST and IGRA were positive in 29.5\%, TST-positive but IGRA-negative results were apparent in 43.4\%. Active TB was diagnosed in twelve HCWs - eight cases were detected during screening and four cases were predicted by IGRA as well as by TST. However, the progression rate in IGRA-positive was higher than in TST-positive HCWs (0.4\% vs. 0.2\%, p-value 0.06$)$.

Conclusions: The TB burden in this cohort was high (129.8 per 100,000 HCWs). However, the progression to active TB after a positive TST or positive IGRA was considerably lower than that reported in literature for close contacts in low-incidence countries. This may indicate that old LTBI prevails in these HCWs.
\end{abstract}

\section{Introduction}

Screening healthcare workers (HCWs) for latent tuberculosis infection (LTBI) and active tuberculosis (TB) disease is fundamental in infection control programmes in hospitals [1]. The tuberculin skin test (TST) was the first method available for detecting LTBI. However, the TST has known limitations, including cross-reactivity with bacillus Calmette-Guérin (BCG) and non-tuberculous mycobacteria (NTM) infections [2]. Recently, new in vitro assays that measure interferon (IFN) $\gamma$ released by sensitised $\mathrm{T}$ cells after stimulation with Mycobacterium tuberculosis antigens have been developed. These tests are more specific than the TST since they use antigens not shared by any of the BCG vaccine strains nor by the more common species of NTM [3]. Interferon- $\gamma$ release assays (IGRAs) also have the advantage of correlating better with surrogate measures of exposure to $M$. tuberculosis [4-6] and have a higher predictive value for

\footnotetext{
*Correspondence: a.nienhaus@uke.uni-hamburg.de

${ }^{3}$ University Medical Centre Hamburg-Eppendorf, Institute for Health Services Research in Dermatology and Nursing, Hamburg, Germany

Full list of author information is available at the end of the article
}

LTBI progression to active TB disease in close contact in low-incidence settings $[7,8]$.

This paper represents a recent analysis of the growing cohort of HCWs tested with IGRA at the University Hospital of Porto, Portugal, between January 2007 and December 2010. In previous papers, TST and IGRA results were compared with respect to risk factors for LTBI $[9,10]$. Now the sample size has been increased from 1,218 to 2,884 HCWs for whom a direct comparison of TST and IGRA is possible. Furthermore, more than three years have passed since the introduction of IGRA screening at the hospital and the predictive value of TST and IGRA for developing active TB in HCWs can be analysed.

\section{Methods}

All workers at the Hospital São João are offered TB screening following the Centers for Disease Control and Prevention (CDC) guidelines [1]. Upon commencement of employment, all workers are examined to exclude active TB disease and to assess the pre-employment status. Depending on the risk assessment, the examination is repeated annually or every other year. HCWs with

\section{() Biomed Central}

(c) 2011 Costa et al; licensee BioMed Central Ltd. This is an Open Access article distributed under the terms of the Creative Commons Attribution License (http://creativecommons.org/licenses/by/2.0), which permits unrestricted use, distribution, and reproduction in any medium, provided the original work is properly cited. 
close patient contacts in the infection and TB wards are considered to be at a high risk, workers with regular patient contacts in the other wards are considered to be at a medium risk and workers with no regular patient contacts and no contact with biological material are considered to be at a low risk. After unprotected contact with an infectious patient, co-worker or material, screening is performed as well.

Since January 2007, screening has been performed using TST and IGRA. A chest X-ray is performed in order to exclude active pulmonary disease when TST is considered positive ( $\geq 10 \mathrm{~mm}$ ), when IGRA is positive and in HCWs with symptoms. BCG vaccination is assessed through the individual vaccination register. If no register is available, vaccination status is verified by scars. BCG vaccination for newborns is mandatory in Portugal and, until January 2000, was repeated depending on risk assessment and TST diameter. Therefore every $\mathrm{HCW}$ is considered to have been vaccinated at least once.

TST is performed by trained personnel following standard procedures. In brief, $0.1 \mathrm{~mL}$ (2 TU) of purified protein derivative (PPD, RT23; Statens Serum Institute, Copenhagen, Denmark) is injected. The TST is administered to the volar side of the forearm of the participants and read 72 to 96 hours after the application. The transverse diameter of the induration is measured by experienced personnel.

Before the TST application, an interview is performed and blood for the IGRA is drawn. For the IGRA, the QuantiFERON-TB ${ }^{\circledR}$ Gold In-Tube Assay (Cellestis Limited, Carnegie, Australia) is used. This whole-blood assay uses overlapping peptides corresponding to ESAT6 , CFP-10 and a portion of the tuberculosis antigen TB7.7 (Rv2654). Stimulation of the antigenic mixture occurs within the tube used to collect blood. Tubes were incubated at $37 \mathrm{C}$ overnight before centrifugation, and INF- $\gamma$ release is measured by ELISA following the protocol of the manufacturer. All assays performed met the manufacturer's quality control standards. The test is considered positive when INF- $\gamma$ is $\geq 0.35$ IU after correction for the negative control. Observers were blinded to the results of the TST results.

A chi-square test was used to compare the frequencies of test results among different groups of participants. For risk factors assessed by ordinal variables, the proportions of positive test results were compared using the chi-square test of trend. A binomial test was used for the comparison of active TB rates and TB predicted rates of TST and IGRA. P $<0.05$ was considered to be statistically significant. Adjusted odds ratios (OR) and 95\% confidence intervals (CI) were calculated for different putative predictive variables using conditional logistic regression.
Data analysis was performed with SPSS, Version 14 (SPSS Inc., Chicago, Illinois). All persons gave their informed consent prior to their inclusion in the study. No additional data was collected for the study purpose only and analysis was performed with anonymous data. Therefore no endorsement by an ethics committee was required.

\section{Results}

The flow chart of the study sample is given in Figure 1. Undetermined results of the IGRA were observed in 5 HCWs $(5 / 2,889)$. A total of $850 \mathrm{HCWs}(29.5 \%)$ was positive in TST and IGRA. Twelve of these HCWs (1.5\%) were diagnosed with active TB. Four HCWs were diagnosed with TB more than three months after the positive TST and IGRA. The characteristics of the study population are given in Table 1 . The cohort is predominantly female $(71.7 \%)$ and the majority were repeatedly vaccinated with BCG (68.2\%). Infection risk was considered moderate for $59.7 \%$ of the cohort. The mean follow-up time for the HCWs was 19 months, SD 5.2 month (no table).

IGRA was positive in $33 \%$ and TST was $10 \mathrm{~mm}$ or higher in $72.9 \%$ of the HCWs (Table 2). The probability of a positive IGRA increased with diameter in TST. However, $42.4 \%$ of the HCWs with a TST of $\geq 20 \mathrm{~mm}$ were negative in IGRA and $11.2 \%$ with a TST of $<5$ $\mathrm{mm}$ were positive in IGRA.

Age is a risk factor for a positive TST ( $\geq 10 \mathrm{~mm})$ as well as a positive IGRA (Table 3). However, the adjusted OR for the IGRA show a more pronounced doseresponse relationship with age than the TST, e.g. OR in HCWs aged 50 or older for positive TST 2.0 and for positive IGRA 2.7 (Table 3). Gender was not associated with TST or IGRA. Repeated BCG vaccination did not

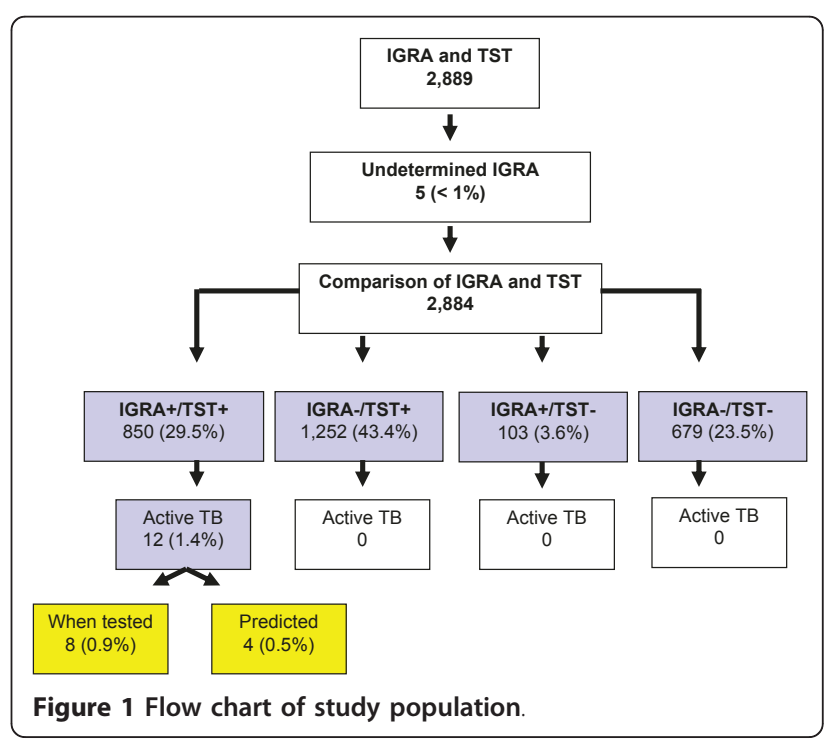




\section{Table 1 Study population for comparison of IGRA with} TST

\begin{tabular}{|c|c|c|}
\hline Age & $\mathrm{N}$ & $\%$ \\
\hline$<25$ years & 301 & 10.4 \\
\hline $25-29$ years & 821 & 28.5 \\
\hline 30-39 years & 791 & 27.4 \\
\hline 40-49 years & 534 & 18.5 \\
\hline$\geq 50$ years & 437 & 15.2 \\
\hline \multicolumn{3}{|l|}{ Gender } \\
\hline Female & 2,068 & 71.7 \\
\hline Male & 816 & 28.3 \\
\hline \multicolumn{3}{|l|}{ BCG vaccination } \\
\hline Only at birth & 917 & 31.8 \\
\hline One additional & 1,022 & 35.4 \\
\hline Two additional & 663 & 23.0 \\
\hline$\geq 3$ additional & 282 & 9.8 \\
\hline \multicolumn{3}{|l|}{ Profession } \\
\hline Administrator & 388 & 13.5 \\
\hline Auxiliaries, cleaning staff & 487 & 16.9 \\
\hline Technicians (radiology, lab, etc.) & 170 & 5.9 \\
\hline Nurses & 1,204 & 41.7 \\
\hline Physicians & 635 & 22.0 \\
\hline \multicolumn{3}{|l|}{ Risk assessment } \\
\hline Low risk & 206 & 7.1 \\
\hline Moderate risk & 1,722 & 59.7 \\
\hline High risk & 956 & 33.1 \\
\hline \multicolumn{3}{|l|}{ Years working in healthcare } \\
\hline Start of work & 400 & 13.9 \\
\hline$<1$ years & 141 & 4.9 \\
\hline $1-5$ years & 787 & 27.3 \\
\hline$>5-10$ years & 465 & 16.1 \\
\hline $10-20$ years & 573 & 19.9 \\
\hline$\geq 20$ years & 518 & 18.0 \\
\hline
\end{tabular}

Table 2 TST diameter by IGRA results

\begin{tabular}{|c|c|c|c|c|c|c|}
\hline \multicolumn{7}{|c|}{ IGRA } \\
\hline \multirow[t]{2}{*}{ TST } & \multicolumn{2}{|c|}{ Negative } & \multicolumn{2}{|c|}{ Positive } & \multicolumn{2}{|c|}{ Total } \\
\hline & $\mathrm{N}$ & Row\% & $\mathrm{N}$ & Row\% & $\mathrm{N}$ & Col\% \\
\hline 0-4 mm & 596 & 88.8 & 75 & 11.2 & 671 & 23.3 \\
\hline 5-9 mm & 83 & 74.8 & 28 & 25.2 & 111 & 3.8 \\
\hline $10-14 \mathrm{~mm}$ & 623 & 73.3 & 227 & 26.7 & 850 & 29.5 \\
\hline $15-19 \mathrm{~mm}$ & 468 & 53.7 & 404 & 46.3 & 872 & 30.2 \\
\hline$\geq 20 \mathrm{~mm}$ & 161 & 42.4 & 219 & 57.6 & 380 & 13.2 \\
\hline$\overline{\text { All }}$ & 1,931 & 67.0 & 953 & 33.0 & 2,884 & 100.0 \\
\hline
\end{tabular}

TST = Tuberculin skin test

IGRA $=$ Interferon $-\gamma$ release assay

Row $\%=\%$ within the TST category

Col\% - Column $\%=\%$ of total falling into a certain TST category

$\mathrm{P}$-value for linear trend $<0.001$
Table 3 Adjusted odds ratios (OR) and 95\% confidence intervals $(\mathrm{Cl})$ for tuberculin skin tests (TST) of $\geq 10 \mathrm{~mm}$ and positive interferon $-\gamma$ release assays (IGRA)

\begin{tabular}{lllllll}
\hline & \multicolumn{2}{l}{ TST $\geq 10 \mathbf{~ m m}$} & \multicolumn{4}{l}{ IGRA-positive } \\
\hline Age & $\mathrm{N}(\%)$ & $\mathrm{OR}$ & $95 \% \mathrm{Cl}$ & $\mathrm{N}(\%)$ & $\mathrm{OR}$ & $95 \% \mathrm{Cl}$ \\
\hline$<25$ years & $223(74.1)$ & 1 & - & $57(18.9)$ & 1 & - \\
\hline $25-29$ years & $532(64.8)$ & 0.6 & $0.5-0.8$ & $204(24.8)$ & 1.3 & $0.93-1.8$ \\
\hline $30-39$ years & $553(69.9)$ & 0.8 & $0.6-1.1$ & $266(33.6)$ & 1.9 & $1.4-2.6$ \\
\hline $40-49$ years & $424(79.4)$ & 1.5 & $1.0-2.1$ & $219(41.0)$ & 2.3 & $1.6-3.3$ \\
\hline$\geq 50$ years & $370(84.7)$ & 2.0 & $1.4-3.0$ & $207(47.4)$ & 2.7 & $1.9-3.9$ \\
\hline Gender & & & & & & \\
\hline Female & 1,487 & 1 & - & $658(31.8)$ & 1 & - \\
& $(71.9)$ & & & & & \\
\hline Male & $615(75.4)$ & 1.2 & $0.97-$ & $295(36.2)$ & 1.1 & $0.9-1.3$ \\
& & & 1.4 & & &
\end{tabular}

\begin{tabular}{|c|c|c|c|c|c|c|}
\hline BCG vaccination & & & & & & \\
\hline Only at birth & $700(76.3)$ & 1 & - & 406 (44.3) & 1 & - \\
\hline One additional & $741(72.5)$ & 1.0 & $0.8-1.2$ & $330(32.3)$ & 0.7 & $0.6-0.9$ \\
\hline Two additional & $460(69.4)$ & 1.0 & $0.7-1.2$ & 165 (24.9) & 0.6 & $0.5-0.7$ \\
\hline 3-10 additional & $201(71.3)$ & 1.0 & $0.8-1.4$ & $52(18.4)$ & 0.4 & $0.3-0.6$ \\
\hline \multicolumn{7}{|l|}{ Profession } \\
\hline Administrator & $302(77.8)$ & 1 & & $156(40.2)$ & 1 & - \\
\hline $\begin{array}{l}\text { Auxiliaries, cleaning } \\
\text { staff }\end{array}$ & $335(68.8)$ & 0.8 & $0.6-1.1$ & $180(37.0)$ & 1.0 & $0.7-1.3$ \\
\hline $\begin{array}{l}\text { Technicians (radiology, } \\
\text { lab, etc.) }\end{array}$ & 137 (80.6) & 1.6 & $1.0-2.5$ & $54(31.8)$ & 1.0 & $0.7-1.5$ \\
\hline Nurses & 864 (71.8) & 1.3 & $\begin{array}{l}0.95- \\
1.8\end{array}$ & 317 (26.3) & 0.8 & $0.6-1.1$ \\
\hline Physicians & $464(73.1)$ & 1.2 & $0.9-1.7$ & $246(38.7)$ & 1.4 & $1.04-1.9$ \\
\hline \multicolumn{7}{|l|}{ Risk assessment } \\
\hline Low risk & $176(85.4)$ & 1 & - & $85(41.3)$ & 1 & - \\
\hline Moderate risk & $\begin{array}{l}1,289 \\
(74.9)\end{array}$ & 0.6 & $\begin{array}{l}0.4- \\
0.91\end{array}$ & $549(31.9)$ & 0.8 & $0.6-1.2$ \\
\hline High risk & $637(66.6)$ & 0.4 & $0.3-0.6$ & $319(33.4)$ & 0.9 & $0.7-1.3$ \\
\hline
\end{tabular}

\section{Years working in}

healthcare*

\begin{tabular}{lllllll}
\hline Start of work & $289(72.3)$ & 1 & - & $112(28.0)$ & 1 & - \\
\hline$<1$ years & $84(59.6)$ & 0.6 & $0.4-$ & $26(18.4)$ & 0.7 & $0.4-1.1$ \\
& & 0.92 & & & \\
\hline $1-5$ years & $502(63.8)$ & 0.7 & $0.5-$ & $211(26.8)$ & 1.1 & $0.8-1.4$ \\
& & 0.91 & & & \\
\hline$>5-10$ years & $324(69.7)$ & 0.9 & $0.7-1.2$ & $154(33.1)$ & 1.4 & $1.06-2.0$ \\
\hline $10-20$ years & $454(79.2)$ & 1.5 & $1.1-2.1$ & $212(37.0)$ & 1.6 & $1.2-2.2$ \\
\hline$\geq 20$ years & $449(86.7)$ & 2.5 & $1.8-3.6$ & $238(45.9)$ & 1.9 & $1.4-2.5$ \\
\hline * Correlation between age and years working in healthcare $r=0.82$. & Separate \\
models were calculated for these variables & & & &
\end{tabular}

influence TST, but was associated with a decreased probability of positive IGRA, e.g. OR $=0.4(95 \%$ CI $0.3-$ $0.6)$ for three or more additional vaccinations. Profession and risk assessment were not associated with TST or IGRA results. The number of years working in healthcare increased the probability of positive TST and 
IGRA. The highest OR (2.5) was observed for TST when working for 20 or more years in healthcare. However, due to high correlation between the variables of age and years working in healthcare, it is not possible to separate both effects.

Discordant TST+/IGRA- combinations are most likely (57.8\%) in HCWs younger than 25 years and less likely in HCWs older than 50 years (40\%). Gender is not associated with discordant TST and IGRA results (Table 4). The probability of TST+/IGRA- discordance increased from $35.8 \%$ in those with BCG vaccination at birth only to $55.7 \%$ in those with three or more repeated vaccinations. Technicians $(52.4 \%)$ and nurses $(48.6 \%)$ had the highest rates of TST+/IGRA- discordant results.
Fifty-seven HCWs (2.0\%) had a history of active TB disease since 2005 and were treated accordingly. Of these, $86 \%$ had a TST of $\geq 10 \mathrm{~mm}$ and $59.6 \%$ a positive IGRA when screened in the scope of this study (Table 5). Eight HCWs had active TB at the time of screening, and progression to active TB within 4 to 24 months of screening occurred in four HCWs. Sensitivity for prevalent and for predicted active TB was $100 \%$ for both TST of $\geq 10 \mathrm{~mm}$ and IGRA. The rate of prevalent and predicted active TB in IGRA-positive HCWs $(0.8 \%$ and $0.4 \%)$ was twice as high as the rates for HCWs with TST of $\geq 10 \mathrm{~mm}(0.4 \%$ and $0.2 \%)$. However, the difference was only statistically significant $(\mathrm{p}=0.008)$ for prevalent active TB. The $\mathrm{p}$-value for the different progression rates was 0.06 (Table 5).

Table 4 Concordant and discordant tuberculin skin test (TST) of $\geq 10 \mathrm{~mm}$ and interferon- $\gamma$ release assay (IGRA) results depending on putative risk factors

\begin{tabular}{|c|c|c|c|c|c|c|c|c|c|}
\hline \multirow[b]{3}{*}{ Age } & \multicolumn{8}{|c|}{ TST/IGRA } & \multirow{3}{*}{$\begin{array}{c}\mathbf{P} \\
<0.001\end{array}$} \\
\hline & \multicolumn{2}{|c|}{ Neg./Neg. } & \multicolumn{2}{|c|}{ Pos./Neg. } & \multicolumn{2}{|c|}{ Neg./Pos. } & \multicolumn{2}{|c|}{ Pos./Pos. } & \\
\hline & $\mathrm{N}$ & $\%$ & $n$ & $\%$ & $n$ & $\%$ & $n$ & $\%$ & \\
\hline$<25$ years & 70 & 23.3 & 174 & 57.8 & 8 & 2.7 & 49 & 16.3 & \\
\hline $25-29$ years & 258 & 31.4 & 359 & 43.7 & 31 & 3.8 & 173 & 21.1 & \\
\hline 30-39 years & 203 & 25.7 & 322 & 40.7 & 35 & 4.4 & 231 & 29.2 & \\
\hline 40-49 years & 93 & 17.4 & 222 & 41.6 & 17 & 3.2 & 202 & 37.8 & \\
\hline$\geq 50$ years & 55 & 12.6 & 175 & 40.0 & 12 & 2.7 & 195 & 44.6 & \\
\hline \multicolumn{10}{|l|}{ Gender } \\
\hline Female & 508 & 24.6 & 902 & 43.6 & 73 & 3.5 & 585 & 28.3 & \\
\hline Male & 171 & 21.0 & 350 & 42.9 & 30 & 3.7 & 265 & 32.5 & 0.078 \\
\hline \multicolumn{10}{|l|}{ BCG vacci. } \\
\hline At birth & 183 & 20.0 & 328 & 35.8 & 34 & 3.7 & 372 & 40.6 & \\
\hline Plus one additional & 245 & 24.0 & 447 & 43.7 & 36 & 3.5 & 294 & 28.8 & \\
\hline Plus two & 178 & 26.8 & 320 & 48.3 & 25 & 3.8 & 140 & 21.1 & \\
\hline Plus 3-10 & 73 & 25.9 & 157 & 55.7 & 8 & 2.8 & 44 & 15.6 & $<0.001$ \\
\hline \multicolumn{10}{|l|}{ Profession } \\
\hline Administrator & 72 & 18.6 & 160 & 41.2 & 14 & 3.6 & 142 & 36.6 & \\
\hline Auxiliaries, cleaning & 133 & 27.3 & 174 & 35.7 & 19 & 3.9 & 161 & 33.1 & \\
\hline Technicians (radiology, lab, etc.) & 27 & 15.9 & 89 & 52.4 & 6 & 3.5 & 48 & 28.2 & \\
\hline Nurses & 302 & 25.1 & 585 & 48.6 & 38 & 3.2 & 279 & 23.2 & \\
\hline Physicians & 145 & 22.8 & 244 & 38.4 & 26 & 4.1 & 220 & 34.6 & $<0.001$ \\
\hline \multicolumn{10}{|l|}{ Risk } \\
\hline Low & 27 & 13.1 & 94 & 45.6 & 3 & 1.5 & 82 & 39.8 & \\
\hline Moderate & 378 & 22.0 & 795 & 46.2 & 55 & 3.2 & 494 & 28.7 & \\
\hline High & 274 & 28.7 & 363 & 38.0 & 45 & 4.7 & 274 & 28.7 & $<0.001$ \\
\hline \multicolumn{10}{|l|}{ Years in healthcare } \\
\hline Start of work & 96 & 24.0 & 192 & 48.0 & 15 & 3.8 & 97 & 24.3 & \\
\hline$<1$ years & 51 & 36.2 & 64 & 45.4 & 6 & 4.3 & 20 & 14.2 & \\
\hline $1-5$ years & 248 & 31.5 & 328 & 41.7 & 37 & 4.7 & 174 & 22.1 & \\
\hline$>5-10$ years & 124 & 26.7 & 187 & 40.2 & 17 & 3.7 & 137 & 29.5 & \\
\hline $10-20$ years & 103 & 18.0 & 258 & 45.0 & 16 & 2.8 & 196 & 34.2 & \\
\hline$\geq 20$ years & 57 & 11.0 & 223 & 43.1 & 12 & 2.3 & 226 & 43.6 & $<0.001$ \\
\hline
\end{tabular}


Table 5 Old, active, and predicted TB by TST and IGRA results

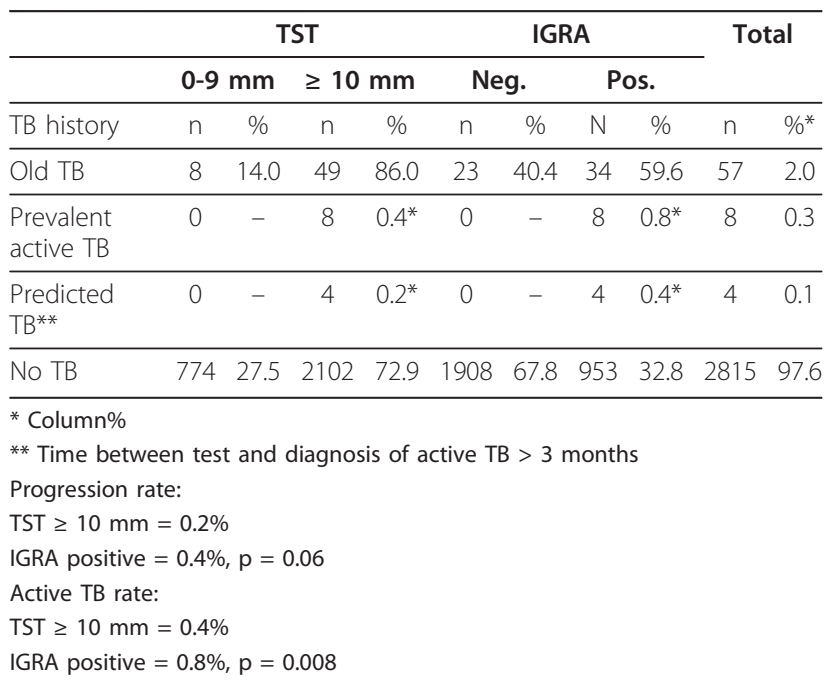

\section{Discussion}

This is the first study to report sensitivity for disease progression in HCWs simultaneously tested with IGRA and TST. However, even though the study sample was huge $(n=2,884)$, calculation of the disease progression rate is based on four cases only. In IGRA-positive HCWs, it was twice as high as in HCWs with TST of $\geq$ $10 \mathrm{~mm}$. However, the difference was not statistically significant. The progression rate we observed was considerably lower than the one observed in close contacts in a German cohort $[7,8]$. Apart from a shorter follow-up period in our study, this indicates that old infections, which have a lower progression risk, prevail in the HCW cohort. As with TST, IGRAs are not able to distinguish old from recent LTBI [11]. In HCWs with a positive IGRA, the likelihood of a recent LTBI can only be assessed by evaluation of the exposure situation within the last months before the IGRA is performed. This should be done from case to case, as the exposure assessment following CDC [1] was not helpful in this endeavour.

Even though the sample size increased from 1,219 to 2,884 compared to our first publication [9], the rates for positive IGRA and TST of $\geq 10 \mathrm{~mm}$ are very similar (32.6\% versus $33.1 \%$ for IGRA and $74.2 \%$ versus $72.9 \%$ for TST of $\geq 10 \mathrm{~mm}$ ). The rate of positive IGRA is considerably higher in Portuguese HCWs than in HCWs from other West-European countries, reflecting the higher TB-incidence in Portugal than in other WestEuropean countries [12-14]. The twelve cases of active TB observed in our population gives rise to a TB incidence rate of 129.8 per $100,000 \mathrm{HCWs}$. The rate is considerably lower than the rate of 191.6 per 100,000 HCWs observed in our previous publication [9]. But the rate is still about four times higher than the annual incidence rate in the general population in Portugal in 2006 of 32/100,000 [9]. This comparison illustrates the beneficial aspects of systematic screening of HCWs in a medium-incidence country and the increased risk of TB infection in HCWs.

In Portugal, BCG vaccination is universal and, until January 2000, was repeated when a person was negative in the TST. This makes it difficult to examine the influence of BCG vaccination on TST or IGRA. Little is currently known about the effect of repeated BCG vaccination on the probability of TST+/IGRA- discordant results $[11,15]$. As was expected, repeated BCG vaccination increased the probability of TST results higher than $10 \mathrm{~mm}$. However, a recent BCG vaccination or a repeated BCG vaccination decreased the probability of a positive IGRA. This might indicate a protective effect of BCG vaccination or may have been caused by the revaccination schema: TST-negative HCWs are revaccinated, inducing a positive TST, without changing the IGRA. Increasing the cut-off for a positive TST from 10 to $15 \mathrm{~mm}$ reduced the number of positive TSTs (72.9 vs. 43.4), but also increased the probability of a positive IGRA not detected by TST $(10.8 \%$ vs. $34.6 \%$, calculated from Table 2). Therefore, this strategy is not suitable to reduce the specificity problems of the TST in a population which is BCG-vaccinated.

Nurses had a higher rate of TST+/QFT - results than physicians (48.6 vs. $38.6 \%$, Table 4 ) but were also tested more often than physicians with TST [9]. This indicates that risk assessment may be biased by using TST. The reason why nurses underwent TST more often than doctors is unknown; but one reasonable assumption is that they were more compliant in earlier contact tracings.

Working in healthcare is a well-known risk factor for TB $[16,17]$. In our data, the probability of a positive IGRA (Table 3) or of TST+/IGRA+ concordance (Table 4) increased with the number of years spent in healthcare. However, this association might be explained by age alone. Surprisingly, neither risk assessment [1] nor profession was associated with TST or with IGRA, or the association observed was in an unexpected direction. In the two European fingerprint studies $[18,19]$, the majority of work-related active TB cases occurred when the infection risk was considered to be low and preventive measures were not in place because TB was not suspected. Rotation of the staff is a further potential explanation for the lack of this association. Therefore risk assessment should be reconsidered.

Screening HCWs for LTBI with TST in our populations had severe shortcomings. The rate of TST of $>10$ $\mathrm{mm}$ was high and was influenced by repeated BCG vaccination, allowing little discrimination between HCWs 
at risk of having or of progressing to active TB. On the contrary, the IGRA was not influenced by BCG vaccination. Therefore our data corroborates the conclusion of other HCW studies $[20,21]$ that the TST is not useful in contact investigations among BCG-vaccinated HCWs, while IGRA may provide additional information for the diagnosis and strategic management of preventive treatment in BCG-vaccinated HCW.

All eight HCWs diagnosed with active TB were positive in both tests. In summary, the IGRA was therefore better than the TST in screening HCWs for LTBI and active TB. Overall, screening of HCWs was successful because of the high number of active TB cases identified through this systematic screening. Future studies should investigate the incidence of new TB infections and the beneficial effect of chemoprevention in these HCWs.

\section{Author details}

${ }^{1}$ Hospital São João, EPE Alameda Professor Hernâni Monteiro, Porto, Portugal. ${ }^{2}$ Faculty of Medicine, Porto University Alameda Professor Hernâni Monteiro, Porto, Portugal. ${ }^{3}$ University Medical Centre Hamburg-Eppendorf, Institute for Health Services Research in Dermatology and Nursing, Hamburg, Germany.

\section{Authors' contributions}

JTC designed the study, performed the physical examinations, and was involved in drafting of the paper. RS was involved in data collection and drafting of the paper. FR was involved in data analysis and drafting of the paper. AN analysed the data and wrote the first draft of the paper. All authors read and approved the final manuscript.

\section{Competing interests}

The authors declare that they do not have any direct or indirect personal relationship, affiliation or association with any party with whom they deal in their day-to-day work that would give rise to any actual or perceived conflict of interest.

Received: 15 March 2011 Accepted: 9 June 2011 Published: 9 June 2011

\section{References}

1. Jensen PA, Lambert LA, lademarco MF, Ridzon R: Guidelines for preventing the transmission of Mycobacterium tuberculosis in Healthcare Settings. MMWR 2005, 54(RR-17):1-141

2. Menzies D: What does tuberculin reactivity after Bacille Calmette-Guerin vaccination tell us? Clin Infect Dis 2000, 31(Suppl. 3):S71-S74.

3. Andersen P, Munk ME, Pollock JM, Doherty TM: Specific immune-based diagnosis of tuberculosis. Lancet 2000, 356:1099-1104.

4. Nahid P, Pai M, Hopewell PC: Advances in the diagnosis and treatment of tuberculosis. Proc Am Thorac Soc 2006, 3:103-110.

5. Diel R, Loddenkemper R, Meywald-Walter K, Gottschalk R, Nienhaus A: Comparative Performance of Tuberculin Skin Test, QuantiFERON-TB-Gold In Tube Assay, and T-Spot.TB Test in Contact Investigations for Tuberculosis. Chest 2009, 135(4):1010-1018.

6. Diel R, Loddenkemper R, Nienhaus A: Evidence based comparison of commercial interferon-gamma release assays for detecting active tuberculosis - a meta-analysis. Chest 2010, 137(4):952-968

7. Diel R, Loddenkemper R, Meywald-Walter K, Niemann S, Nienhaus A: Predictive value of a whole blood IFN-c assay for the development of active TB disease. Am J Respir Crit Care Med 2008, 177:1164-1170.

8. Diel R, Loddenkemper R, Niemann S, Meywald-Walter K, Nienhaus A: Negative and positive predictive value of a whole-blood Interferon- $\gamma$ Release Assay for developing active tuberculosis - An Update. Am J Respir Crit Care Med 2011, 183:88-95

9. Torres Costa J, Sá R, Cardoso MJ, Silva R, Ferreira J, Ribeiro C, Miranda M, Plácido JL, Nienhaus A: Tuberculosis screening in Portuguese healthcare workers using the tuberculin skin test and the interferon-gamma release assay. Eur Respir J 2009, 34:1423-8.

10. Torres Costa J, Silva R, Cardoso MJ, Nienhaus A: Results of five-year systematic screening for latent tuberculosis infection in healthcare workers in Portugal. J Occup Med Toxicol 2010, 5:22.

11. Nienhaus A, Schablon A, Diel R: Interferon- $\gamma$ release assay for the diagnosis of latent TB infection - analysis of discordant results, when compared to the tuberculin skin test. PLOS ONE 2008, 3(7):e2665.

12. Schablon A, Beckmann G, Harling M, Diel R, Nienhaus A: Prevalence of latent tuberculosis infection among healthcare workers in a hospital for pulmonary diseases. J Occup Med Toxicol 2009, 4:1

13. Schablon A, Harling M, Diel R, Nienhaus A: Risk of latent TB infection in individuals employed in the healthcare sector in Germany: a multicentre prevalence study. BMC Infect Dis 2010, 10:107.

14. Tripodi D, Brunet-Court, Nael V, Audrain M, Chailleux E, Germaud P, Naudin F, Muller JY, Bourrut-Lacouture M, Durand-Perdriel MH, Gordeeff C, Guillaumin G, Houdebine M, Raffi F, Boutoille D, Biron C, Potel G, Roedlich C, Geraut C, Schablon A, Nienhaus A: Evaluation of the tuberculin skin test and the interferon-gamma release assay for TB screening in French healthcare workers. J Occup Med Toxicol 2009, 4:30.

15. Farhat M, Greenaway, Pai M, Menzies D: False-positive tuberculin skin tests: what is the absolute effect of BCG and non-tuberculous mycobacteria? Int J Tuberc Lung Dis 2006, 10(11):1192-1204.

16. Menzies $D$, Joshi R, Pai M: Risk of tuberculosis infection and disease associated with work in health care settings. Int I Tuberc Lung Dis 2007, 11(6):593-605

17. Seidler A, Nienhaus A, Diel R: Review of epidemiological studies on the occupational risk of tuberculosis in low-incidence areas. Respiration 2005, 72(4):431-446.

18. Diel R, Seidler A, Nienhaus A, Rusch-Gerdes S, Niemann S: Occupational risk of tuberculosis transmission in a low incidence area. Respir Res 2005, 6(1):35-45.

19. De Vries G, Sebek MM, Lambregts-van Weezenbeek CS: Healthcare workers with tuberculosis infected during work. Eur Respir J 2006, 28(6):1216-21.

20. Nienhaus A, Schablon A, Siano B, le Bacle C, Diel R: Evaluation of the interferon-gamma release assay in healthcare workers. Int Arch Occup Environ Health 2008, 81:295-300.

21. Ringshausen FC, Schlosser S, Nienhaus A, Schablon A, SchultzeWerninghaus $\mathrm{G}$, Rohde $\mathrm{G}$ : In-hospital contact investigation among health care workers after exposure to smear-negative tuberculosis. J Occup Med Toxicol 2009, 4(1):11.

doi:10.1186/1745-6673-6-19

Cite this article as: Torres Costa et al: Screening for tuberculosis and prediction of disease in Portuguese healthcare workers. Journal of Occupational Medicine and Toxicology 2011 6:19.

\section{Submit your next manuscript to BioMed Central and take full advantage of:}

- Convenient online submission

- Thorough peer review

- No space constraints or color figure charges

- Immediate publication on acceptance

- Inclusion in PubMed, CAS, Scopus and Google Scholar

- Research which is freely available for redistribution 